\title{
Accuracy of Transcranial Doppler Ultrasound Compared with Magnetic Resonance Angiography in the Diagnosis of Intracranial Artery Stenosis
}

\author{
Sandip Kumar Jaiswal ${ }^{1}$ Yan Fu-ling ${ }^{1}$ Lihua $\mathrm{Gu}^{1} \quad$ Renardo Lico $^{1} \quad$ Fu Changyong ${ }^{1} \quad$ Angela Paula \\ ${ }^{1}$ Department of Neurology, Zhongda Hospital Affiliated to \\ Southeast University, Nanjing, China \\ Address for correspondence Yan Fu-Ling, Department of Neurology, \\ Zhongda Hospital Affiliated to Southeast University, Dingjiaqiao 87, \\ Nanjing, China (e-mail: yanfuling218@163.com).
}

J Neurosci Rural Pract 2019;10:400-404

\begin{abstract}
Background One of the most common causes of acute cerebral infarction (ACl) is intracranial artery stenosis (ICAS). The goal of our study was to evaluate the accuracy of transcranial Doppler (TCD) compared with magnetic resonance angiography (MRA) for diagnosing ICAS in patients with ACI.

Materials and Methods Consecutive patients presenting with $\mathrm{ACl}$ to the neurology department underwent both MRA and TCD examination within 6 hours of difference. To calculate the agreement between the results of MRA and TCD, kappa coefficient test was used. Sensitivity, specificity, and positive and negative TCD predictive values have been calculated in comparison with MRA.

Results A total of 115 patients was included. There were 77 males (66.95\%) and 38 females (33.04\%). The mean age of patients was $68.32 \pm 10.66$ years (range $29-80$ ).

Keywords

- acute cerebral infarction

- intracranial artery stenosis

- transcranial Doppler ultrasound

- magnetic resonance angiography The agreement between TCD and MRA in detecting stenosis was 0.56 for anterior circulation artery (ACA), and 0.40 for posterior circulation artery. For the detection of ICAS, sensitivity, specificity, positive predictive value, and negative predictive values were $85.9,90.0,98.2$, and $50.0 \%$ for anterior cerebral artery and $73.5,86.7,96.2$, and $40.0 \%$ for posterior cerebral artery, respectively.

Conclusions Moderate agreement of anterior circulation stenosis and fair agreement for posterior circulation stenosis was found between TCD and MRA in the evaluation of ICAS. In anterior circulation, the diagnostic accuracy of TCD is higher compared with the posterior circulation.
\end{abstract}

\section{Introduction}

Imaging tests, such as transcranial Doppler (TCD), magnetic resonance angiography (MRA), computed tomography angiography (CTA), and digital subtraction angiography (DSA), are available for intracranial artery stenosis (ICAS) diagnosis. ${ }^{1}$ Among these methods, TCD is a comparatively affordable, noninvasive, portable, and technically simpler tool of a diagnostic method for diagnosing ICAS. ${ }^{2}$ Although this technique is widely available and very affordable, due to its high operator-dependent nature and limited scope of application, its diagnostic accuracy is generally debated in patients with sufficient acoustic windows. ${ }^{3}$ Although the accuracy of TCD in calculating the severity of stenosis differs from reporting studies, it is advantageous to find out ICAS because it provides real-time information about the velocity of blood flow compared with static imaging methods, such as CTA or MRA. ${ }^{4,5}$ MRA is another noninvasive method used to assess the status of intracranial arteries and circle of Willis and has shown a higher sensitivity and specificity to determine ICAS than conventional DSA. ${ }^{6} \mathrm{~A}$ few studies have focused on the correlation between TCD and MRA to the diagnosis of ICAS.7 The goal of our study was to evaluate the accuracy of TCD 
compared with MRA for diagnosing ICAS in patients with acute cerebral infarction (ACI).

\section{Materials and Methods}

We retrospectively examined consecutive patients who presented with symptoms of ACI between April 1, 2017 and April 30, 2018. All patients were collected from the Affiliated Zhongda Hospital of Southeast University. Eligible patients have been included in this analysis if they fulfill the following criteria. Inclusion criteria: (1) patients hospitalized with clinical sign of ACI within 24 hours of onset, (2) age between 18 and 80 years, and (3) clinical examination and neuroimaging showed ACI; Exclusion criteria: (1) computed tomography (CT) scan of the head demonstrated intracerebral hemorrhage, (2) insufficient acoustic bone windows in TCD, and (3) contraindication for MRA.

All patients underwent CT scan (SOMATOM sensation; Germany) head to exclude intracerebral hemorrhage or tumors.

The basic examinations included a physical examination, a medical history, routine blood tests, chest X-ray, electrocardiogram, lipid profile, coagulation profile, and thyroid function test. Patients have also been screened for infectious disease. Patient's baseline characteristics were recorded, including age, gender, medical history, and smoking/alcohol consumption history. The following risk factors were considered: hypertension, which is characterized those receiving antihypertensive drugs with systolic blood pressure (BP) $\geq 140 \mathrm{~mm} \mathrm{Hg}$ or diastolic BP $\geq 90 \mathrm{~mm}$ Hg more than once. ${ }^{8}$ Diabetes mellitus, including taking antidiabetic medication or having fasting blood glucose level $>126 \mathrm{mg} / \mathrm{dL}$ ( $\geq 7.0 \mathrm{mmol} / \mathrm{L}){ }^{9}$ Hyperlipidemia, including on lipid-lowering agent, total cholesterol level of $>220 \mathrm{mg} / \mathrm{dL}$ (5.69 mm) or low-density lipoprotein $\geq 130 \mathrm{mg} / \mathrm{dL} .{ }^{10}$ In addition, the current smoker was defined as a patient who continuously smoked 1 cigarette per day for 6 months, and heavy drinker defined as drinking $>2$ units per day on average for men or $>1$ unit per day on average for women. Atrial fibrillation has also been included in the analysis as risk points.

This study protocol is in line with the Helsinki Declaration and was approved by the Southeast University Ethics Committee. Written informed consents were taken from all patients.

\section{Transcranial Doppler Finding of Intracranial Artery Stenosis}

Immediately after admission, all eligible patients received a TCD bedside exam, conducted by an experienced sonographer who was not familiar with the clinical report of the patients in accordance with a standardized rapid (lasting $<15$ minutes) insonation protocol. ${ }^{11}$ Our department routinely uses $2 \mathrm{MHz}$ TCD probe to identify ICAS and cerebral arteries blood flow pattern through acoustic windows. The transtemporal approach detected the anterior cerebral artery (ACA), middle cerebral artery (MCA), terminal internal carotid artery (TICA), and posterior cerebral artery (PCA), and the occipital window detected the vertebral artery (VA) and basilar artery (BA). For exposure to different arteries, the insonation depth was as follows: 65 to $72 \mathrm{~mm}$ for ACA, 50 to $65 \mathrm{~mm}$ for MCA, 65 to $75 \mathrm{~mm}$ for TICA, $65 \mathrm{~mm}$ for PCA, 62 to $70 \mathrm{~mm}$ for VA, and 85 to $95 \mathrm{~mm}$ for BA. For each artery, TCD recorded certain parameters that are directly measured, such as peak systolic velocity (PV), and end-diastolic velocity (EDV). The blood flow direction and the waveform characteristics are evaluated. In addition, the derived parameters include mean flow velocity (MFV), the pulsatility index (PI), and resistivity index (RI). PI is a common measure to describe the shape of the waveform of the signal and is calculated using PI $=(\mathrm{PV}-\mathrm{EDV}) / \mathrm{MFV}$ formula. $\mathrm{RI}$ is a peripheral flow resistance measurement and is calculated using the $\mathrm{RI}=(\mathrm{PV}-\mathrm{EDV}) / \mathrm{PV}$ formula. The purpose of the probe was to achieve the highest frequency of Doppler at each depth.

Anterior circulation stenosis was diagnosed if one of the following characteristics has been present: (1) acceleration of flow stenosis was diagnosed if the PV was higher than $120 \mathrm{~cm} / \mathrm{s}$ at a circumscribed insonation depth. To differentiate high-velocity flow from stenosis due to hemispheric hyperperfusion, a stenotic lesion was diagnosed only under the following conditions: (i) if changes in flow speed were "circumscribed" (maximum changes in flow speed were limited to short segment); (ii) if flow distal segment flow velocity (FV) (ipsilateral terminal internal carotid, middle cerebral, or ACA) became blunted; and (iii) if there was an abnormal spectrum (i.e., circumscribed turbulence or musical murmur). (2) Side-to-side differencestenosis was diagnosed if the velocity of contralateral artery was higher by $30 \% .^{12}$

Posterior circulation stenosis was diagnosed using maximum MFV obtained from the PCA, VA, and BA with a 4-second spectral Doppler data acquisition sweep. Stenosis of more than $\geq 50 \%$ was diagnosed when MFV was more than $80 \mathrm{~cm} / \mathrm{s}^{13}$ and when stenotic-to-normal MFV ratio was $\geq 2$, with side-to-side differences $>20 \%$ and circumscribed turbulence or musical murmur at the site of the stenosis..$^{14,15}$

\section{MRA Examination}

MRA was performed on a 3.0 Tesla Magnet system (Philips) with axial three-dimensional time of flight MRA of the circle of Willis was obtained $\left(\mathrm{TR}=15.0 \mathrm{~ms}, \mathrm{TE}=3.45 \mathrm{~ms}\right.$, flip angle $=25, \mathrm{~N}_{\mathrm{EX}}=$ 1 , field of view $=242 \times 242 \mathrm{~mm}$, matrix size $512 \times 512$, 24 slices $\times 3$ sections, slice thickness $1 \mathrm{~mm}$ ) in patients with no evidence of intracranial bleeding and no contraindication for MRA. Source images and maximum intensity projection rendering have been obtained. The reduction in luminal diameter of the residual lumen of at least 50\% applying the method explained in the Warfarin-Aspirin for Symptomatic Intracranial Disease Study was considered to represent a significant ICAS. ${ }^{16}$ Two neuroradiologists without knowledge of the clinical findings independently interpreted MRA studies and agreement was obtained for the final analysis.

\section{Statistical Analysis}

SPSS software (version 20, SPSS Inc., Chicago, Illinois, United States) was used for data storage and analysis. The descriptive data were shown as the mean \pm standard deviation or median 
(range). To calculate the agreement between the results of the MRA and TCD, the kappa coefficient test was used. Sensitivity, specificity, and positive and negative TCD predictive values have been calculated in comparison with MRA.

\section{Results}

In total, 150 of ACI patients were involved in the present study. Of these, 20 patients were excluded due to an insufficient acoustic bone window during TCD examination, 6 patients due to contraindication for MRA (metallic impl ants, pacemaker in the heart), and 9 patients due to intracerebral hemorrhages complication after intravenous thrombolytic therapy. In this study, the remaining 115 patients were included. There were 77 males (66.95\%) and 38 females (33.04\%). Mean age of patients was $68.32 \pm 10.66$ years (range 29-80). Median National Institute of Health Stroke Scale score was 4, range, 2 to 24 points. Median modified Rankin Scale score was 3 , range, 3 to 5 points. According to the National Institute of Neurological Disease and the protocol for stroke trial, 50 patients eligible for intravenous thrombolytic treatment have been treated. ${ }^{17}$ After 24 hours, the CT scans of head was done which showed 9 patients with intracerebral hemorrhages complications following intravenous thrombolytic treatment. The demographic characteristic and vascular risk factors for ACI patients are shown in - Table 1. All ACI patients were examined with TCD and MRA within 6 hours of the difference. The interval between MRA and TCD performance was $6 \pm 1.0$ hours. The TCD was performed before MRA in 15 patients and the MRA was performed before TCD in 100 patients. Length of time between TCD and MRA was 30 to 150 minutes. The TCD and MRA findings of ICAS are summarized in - Table 2 .

Kappa coefficient values calculated to determine the agreement between TCD and MRA for assessment of ACA was 0.56 (moderate agreement), for PCA it was 0.40 (fair agreement).

Table 1 Demographic characteristics and vascular risk factors of the study population $(n=115)$

\begin{tabular}{|l|l|}
\hline Variables & All patients $(\boldsymbol{n}=\mathbf{1 1 5})$ \\
\hline Age, y (mean \pm SD) & $66.32 \pm 10.66$ \\
\hline Gender, male & $77(67.0 \%)$ \\
\hline HTN & $84(73.0 \%)$ \\
\hline DM & $36(31.3 \%)$ \\
\hline AF & $22(19.1 \%)$ \\
\hline Hyperlipidemia & $4(3.5 \%)$ \\
\hline Smoking & $50(43.5 \%)$ \\
\hline Alcohol & $24(20.9 \%)$ \\
\hline Previous stroke or TIA & $25(21.7 \%)$ \\
\hline Median admission NIHSS, range & $2-24$ \\
\hline Median admission mRS, range & $3-5$ \\
\hline
\end{tabular}

Abbreviations: AF, atrial fibrillation; DM, diabetes mellitus; HTN, hypertension; mRS, modified Rankin Scale; NIHSS, National Institute of Health Stroke Scale; SD, standard deviation; TIA, transient ischemic attack.
The sensitivity, specificity, positive predictive value, and negative predictive value for the detection of ICAS were 85.9, 90.0, 98.2, and 50.0\% for anterior circulation and 73.5, 86.7, 96.2, and $40.0 \%$ for posterior circulation on TCD in comparison to MRA, and are presented in - Tables 3 and $\mathbf{4}$. The diagnostic accuracy of TCD in anterior circulation is higher in comparison to posterior circulation. MRA showing stenosis in MCA and BA along with TCD findings in the respective arteries are shown in - Fig. 1.

\section{Discussion}

In Asia, ICAS is considered a major cause of stroke. ${ }^{18}$ To detect ICAS, several noninvasive diagnostic tools were used. MRA, CTA, and TCD methods are commonly used. TCD is one of the most frequently used tests due to its simplicity and affordability; moreover, it permits monitoring of bedside in critical condition. ${ }^{19}$ It also provides further information on the intracranial circulation's hemodynamics status in real time. Nonetheless, TCD has some limitation. For example, in our study, we found that $20(13.3 \%)$ patients have a lack of sufficient acoustic window; however, it also depends on the operator. The failure rate increases with age and also in women due to the higher prevalence of temporal hyperostosis.

Table 2 Number of stenosis in anterior and posterior circulation arteries as detected by TCD and MRA

\begin{tabular}{|l|l|l|}
\hline Location & TCD & MRA \\
\hline Anterior circulation & 56 & 64 \\
\hline Posterior circulation & 26 & 34 \\
\hline
\end{tabular}

Abbreviations: MRA, magnetic resonance angiography; TCD, transcranial Doppler.

Table 3 Sensitivity, specificity, and positive and negative predictive values of transcranial Doppler for detection of ICAS in comparison with magnetic resonance angiography in anterior circulation stenosis

\begin{tabular}{|l|l|}
\hline Parameter & Value \\
\hline Sensitivity, \% & 85.9 \\
\hline Specificity, \% & 90.0 \\
\hline PPV, \% & 98.2 \\
\hline NPV, \% & 50.0 \\
\hline
\end{tabular}

Abbreviations: ICAS, intracranial artery stenosis; NPV, negative predictive value; PPV, positive predictive value.

Table 4 Sensitivity, specificity, and positive and negative predictive values of transcranial Doppler for detection of ICAS in comparison with magnetic resonance angiography in posterior circulation stenosis

\begin{tabular}{|l|l|}
\hline Parameter & Value \\
\hline Sensitivity, \% & 73.5 \\
\hline Specificity, \% & 86.7 \\
\hline PPV, \% & 96.2 \\
\hline NPV, \% & 40.0 \\
\hline
\end{tabular}

Abbreviations: ICAS, intracranial artery stenosis; NPV, negative predictive value; PPV, positive predictive value. 

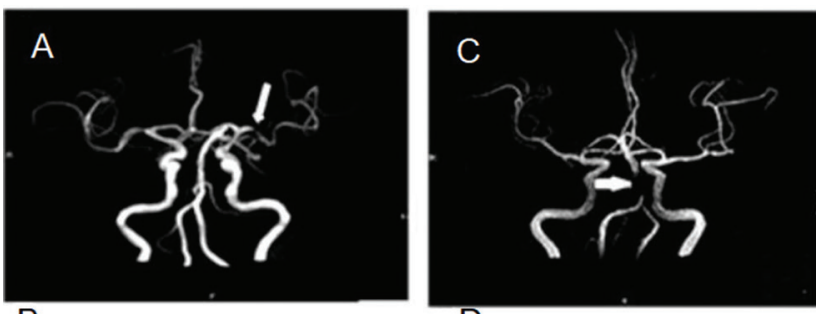

B

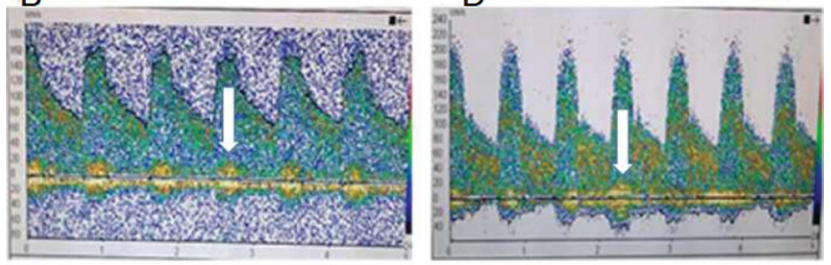

Fig. 1 A 65-year-old female with left middle cerebral artery ischemic stroke who presented with right-sided hemiparesis. (A) MRA showed stenosis of left MCA (white arrow). (B) TCD showed markedly elevated flow velocity $(181 / 81 \mathrm{~cm} / \mathrm{s})$ and musical murmurs (white arrow) in the left MCA. A 72-year-old female with brainstem infarction who presented with dizziness and unstable to walk (C) MRA showed stenosis of BA (white arrow). (D) TCD showed markedly elevated blood flow velocity $(220 / 90 \mathrm{~cm} / \mathrm{s})$ and musical murmurs (white arrow) in the BA.

RI is a TCD parameter that is used to measure the peripheral flow resistance and is calculated using the $\mathrm{RI}=(\mathrm{PSV}-\mathrm{EDV}) / \mathrm{PSV}$ formula. Any value below 0.75 is normal. RI value above 0.8 indicates increased downstream resistance. The changes in RI reflect similar patterns of disease as seen in an abnormal PI. ${ }^{20}$ It was observed that RI also represents a good estimate of high intracranial pressure (ICP) in various intracranial pathologies. However, the RI index is less sensitive to ICP when compared with PI. There is significant variation in patients when measured at a different time. The velocity of MCA flow varies by approximately $10 \%$ cyclically. ${ }^{21}$ The FV of MCA varies cyclically by approximately $10 \%{ }^{22}$ Side-to-side variation was evaluated and the difference of more than $14 \%$ should be considered abnormal. ${ }^{23}$ Day-to-day variation in $95 \%$ of people should be less than $10 \mathrm{~cm} / \mathrm{s}$. The variability between observers was reported to be around $7.5 \%$ on the same day, and approximately $13 \%$ on different days. ${ }^{24}$

DSA is considered the gold standard for extracranial artery stenosis and ICAS to evaluate stroke patients. ${ }^{25}$ It is, however, invasive, costly, and associated with major "periprocedural" complications. ${ }^{26}$ MRA delivers angiogram-like images that multiple planes can be by a three-dimensional post processing. ${ }^{27}$ Time-of-flight MRA generates angiogram-like images in multiple planes by 3-dimensional postprocessing. In addition, the combination of TCD and MRA is more reliable than DSA alone in the identification of MCA stenosis, ${ }^{28}$ and helpful in defining the border zone infarction stroke mechanism in carotid stenosis. ${ }^{29}$

Our research demonstrated the accuracy of TCD in diagnosing ICAS in patients with $\mathrm{ACI}$ and showed that the diagnostic accuracy of TCD is higher, particularly in anterior circulation where sensitivity is $85.9 \%$ and specificity is $90.0 \%$. However, the accuracy of TCD in the diagnosis of posterior circulation is less than that of anterior circulation, because of awkward insonation angles and high frequency of morphological variations in the posterior circulation especially in BA and VA. In recent years, several studies have published the accuracy of TCD compared with MRA in diagnosing ICAS in ACI ${ }^{7,30}$ As a noninvasive and safe method, TCD can detect not only the site of stenosis or occlusion of intracranial arteries but also the degree of the lesion. ${ }^{31}$

Our research showed moderate agreement between TCD and MRA for evaluation of anterior circulation stenosis. Similar results were previously obtained by Navarro et al, ${ }^{32}$ who compared 573 patients referred for TCD with contrast angiography or MRA in a row. They calculated accuracy parameters for TCD were as follows: sensitivity $=90 \%$, specificity $=83 \%$. Our research showed fair agreement for the diagnosis of posterior circulation stenosis between TCD and MRA. Our results in the posterior circulation are consistent with the reports by Ghorbani et $\mathrm{al}^{33}$ and de Bray et $\mathrm{al},{ }^{34}$ who found that TCD has a lower sensitivity but high ICAS diagnostic specificity. This can probably be explained by an anatomical variation of the posterior circulation, and for TCD insonation it can be difficult to locate. ${ }^{35}$ Our findings are inconsistent with Gujjar et al, ${ }^{36}$ who reported a poor correlation between TCD and MRA in acute ischemic stroke patients. In the previous study, TCD studies were performed within 5 days of the onset of stroke. The mean time interval between MRA and TCD was $6 \pm 1.0$ hours in our study. A long time delay between TCD and MRA can provide ample time for the dissolution of thrombus propagation, consideration of discrepancies between the studies, and guaranteed minimum biological modification during this period.

Our study has some limitation. First, a single-center study was conducted. Second, the sample size was very small. Third, some patients were excluded because they did not have an acoustic bone window and others had contraindications for MRA. Fourth, TCD is an operator-dependent technique that requires considerable experience in intracranial arterial anatomy and understanding.

\section{Conclusion}

In the evaluation of ICAS, moderate agreement was found for anterior circulation stenosis and fair agreement for posterior circulation stenosis between TCD and MRA. In anterior circulation, the diagnostic accuracy of TCD is higher compared with posterior circulation. TCD is a relatively cheap, noninvasive, portable, and technically easier tool. The validation of intracranial vascular anatomy with TCD is less than MRA, especially in the peripheral intracranial circulation. TCD provides information on the blood flow velocities of the intracranial blood vessels in real time, whereas MRA only provides anatomical details.

\section{Funding}

None.

\section{Conflict of Interest}

None declared. 


\section{References}

1 Hussain MS, Gupta R. Quantification and assessment of extracranial and intracranial occlusive disease. J Vasc Interv Neurol 2008;1(1):14-15

2 Leng X, Wong KS, Liebeskind DS. Evaluating intracranial atherosclerosis rather than intracranial stenosis. Stroke 2014;45(2):645-651

3 Kalanuria A, Nyquist PA, Armonda RA, Razumovsky A. Use of transcranial Doppler (TCD) ultrasound in the neurocritical care unit. Neurosurg Clin N Am 2013;24(3):441-456

4 Feldmann E, Wilterdink JL, Kosinski A, et al; Stroke Outcomes and Neuroimaging of Intracranial Atherosclerosis (SONIA) Trial Investigators. The Stroke Outcomes and Neuroimaging of Intracranial Atherosclerosis (SONIA) trial. Neurology 2007;68(24):2099-2106

5 Guan J, Zhou Q, Ouyang H, Zhang S, Lu Z. The diagnostic accuracy of TCD for intracranial arterial stenosis/occlusion in patients with acute ischemic stroke: the importance of time interval between detection of TCD and CTA. Neurol Res 2013;35(9):930-936

6 Riles TS, Eidelman EM, Litt AW, Pinto RS, Oldford F, Schwartzenberg GW. Comparison of magnetic resonance angiography, conventional angiography, and duplex scanning. Stroke 1992;23(3):341-346

7 Kenton AR, Martin PJ, Abbott RJ, Moody AR. Comparison of transcranial color-coded sonography and magnetic resonance angiography in acute stroke. Stroke 1997;28(8):1601-1606

8 An SA, Lee HB, Kim Y, et al. Plasma total homocysteine level is associated with the pulsatility index of cerebral arteries in lacunar infarction. Yonsei Med J 2013;54(4):819-824

9 Shibazaki K, Kimura K, Fujii S, Sakai K, Iguchi Y. Brain natriuretic peptide levels as a predictor for new atrial fibrillation during hospitalization in patients with acute ischemic stroke. Am J Cardiol 2012;109(9):1303-1307

10 Li Y, Cai Y, Zhao M, Sun J. Risk factors between intracranial-extracranial atherosclerosis and anterior-posterior circulation stroke in ischaemic stroke. Neurol Res 2017;39(1):30-35

11 Chernyshev OY, Garami Z, Calleja S, et al. Yield and accuracy of urgent combined carotid/transcranial ultrasound testing in acute cerebral ischemia. Stroke 2005;36(1):32-37

12 Alexandrov AV, Sloan MA, Wong LK, et al; American Society of Neuroimaging Practice Guidelines Committee. Practice standards for transcranial Doppler ultrasound: part I-test performance. J Neuroimaging 2007;17(1):11-18

13 Stroke Outcomes and Neuroimaging of Intracranial Atherosclerosis (SONIA) Trail Investigators. Stroke outcome and neuroimaging of intracranial atherosclerosis (SONIA): design of a prospective, multicenter trial of diagnostic tests. Neuroepidemiology 2004;23(1-2):23-32

14 Felberg RA, Christou I, Demchuk AM, Malkoff M, Alexandrov AV. Screening for intracranial stenosis with transcranial Doppler: the accuracy of mean flow velocity thresholds. J Neuroimaging 2002;12(1):9-14

15 Baumgartner RW, Mattle HP, Schroth G. Assessment of $>\mid=50 \%$ and $<50 \%$ intracranial stenoses by transcranial color-coded duplex sonography. Stroke 1999;30(1):87-92

16 Samuels OB, Joseph GJ, Lynn MJ, Smith HA, Chimowitz MI. A standardized method for measuring intracranial arterial stenosis. AJNR Am J Neuroradiol 2000;21(4):643-646

17 Wahlgren N, Ahmed N, Eriksson N, et al; Safe Implementation of Thrombolysis in Stroke-MOnitoring STudy Investigators. Multivariable analysis of outcome predictors and adjustment of main outcome results to baseline data profile in randomized controlled trials: Safe Implementation of Thrombolysis in Stroke-MOnitoring STudy (SITS-MOST) Stroke 2008;39(12):3316-3322
18 Oh HG, Chung PW, Rhee EJ. Increased risk for intracranial arterial stenosis in subjects with coronary artery calcification. Stroke 2015;46(1):151-156

19 Alexandrov AV, Demchuk AM, Wein TH, Grotta JC. Yield of transcranial Doppler in acute cerebral ischemia. Stroke 1999;30(8):1604-1609

20 White H, Venkatesh B. Applications of transcranial Doppler in the ICU: a review. Intensive Care Med 2006;32(7):981-994

21 Krejza J, Mariak Z, Walecki J. Szydlik P, Lewko J, Ustymowicz A. Transcranial color Doppler sonography of basal cerebral arteries in 182 healthy subjects: age and sex variability and normal reference values for blood flow parameters. AJR. Am J Roentgenol 1999;172(1):213-218

22 Giller CA, Lam M, Roseland A. Periodic variations in transcranial Doppler mean velocities. J Neuroimaging 1993;3(3):160-162

23 Tong DC, Albers GW, Normal values. In: Babikian V, Wechsler LR, eds. Transcranial Doppler Ultrasonography. Boston: Butterworth- Heinemann;1999:33-46

24 Maeda $\mathrm{H}$, Etani $\mathrm{H}$, Handa N, et al. A validation study on the reproducibility of transcranial Doppler velocimetry. Ultrasound Med Biol 1990;16(1):9-14

25 Hill MD, Demchuk AM, Frayne R. Noninvasive imaging is improving but digital subtraction angiography remains the gold standard. Neurology 2007;68(24):2057-2058

26 Bendszus M, Koltzenburg M, Burger R. Warmuth-Metz M, Hofmann E, Solymosi L. Silent embolism in diagnostic cerebral angiography and neurointerventional procedures: a prospective study. Lancet 1999;354(9190):1594-1597

27 Sawada M, Yano H, Shinoda J, Funakoshi T, Kumagai M. Symptomatic middle cerebral artery stenosis and occlusion: comparison of three-dimensional time-of-flight magnetic resonance angiography with conventional angiography. Neurol Med Chir (Tokyo) 1994;34(10):682-685

28 Rother J, Schwartz A, Wentz KU, Rautenberg W, Hennerici M. Middle cerebral artery stenosis: assessment by magnetic resonance angiography and transcranial Doppler ultrasound/ magnetic resonance imaging correlates. Cerebrovasc Dis 2004; 17:287-295

29 Kumral E, Bayülkem G, Sağcan A. Mechanisms of single and multiple borderzone infarct: transcranial Doppler ultrasound/ magnetic resonance imaging correlates. Cerebrovasc Dis 2004;17(4):287-295

30 Anzola GP, Gasparotti R, Magoni M, Prandini F. Transcranial Doppler sonography and magnetic resonance angiography in the assessment of collateral hemispheric flow in patients with carotid artery disease. Stroke 1995;26(2):214-217

31 Sarkar S, Ghosh S, Ghosh SK, Collier A. Role of transcranial Doppler ultrasonography in stroke. Postgrad Med J 2007;83(985):683-689

32 Navarro JC, Mikulik R, Garami Z, Alexandrov AV. The accuracy of transcranial Doppler in the diagnosis of stenosis or occlusion of the terminal internal carotid artery. J Neuroimaging 2004;14(4):314-318

33 Ghorbani A, Ashtari F, Fatehi F. The assessment value of transcranial Doppler sonography versus magnetic resonance angiography in vertebrobasilar stroke. J Res Med Sci 2010;15(3):133-139

34 de Bray JM, Missoum A, Dubas F, Emile J, Lhoste P. Detection of vertebrobasilar intracranial stenoses: transcranial Doppler sonography versus angiography. J Ultrasound Med 1997;16(3):213-218

35 Wijman CA, Babikian VL, Matjucha IC, et al. Cerebral microembolism in patients with retinal ischemia. Stroke 1998;29(6):1139-1143

36 Gujjar AR, William R, Jacob PC, Jain R, Al-Asmi AR. Transcranial Doppler ultrasonography in acute ischemic stroke predicts stroke subtype and clinical outcome: a study in Omani population. J Clin Monit Comput 2011;25(2):121-128 